\title{
LA POLÍTICA FISCAL Y MONETARIA EN COLOMBIA: UN ANÁLISIS EXPERIMENTAL DESDE LA TOMA COORDINADA DE DECISIONES
}

\author{
Por: Raúl Rodríguez Luna ${ }^{1}$ - Jairo Mendoza ${ }^{2}$-Erik Romo Salas ${ }^{3}$
}

\section{RESUMEN}

En este documento se discuten los resultados de un ejercicio de simulación a partir de métodos de la economía experimental y modelado con elementos de la teoría de juegos, en el cual se formuló un juego secuencial que permitió encontrar equilibrios de Nash perfectos en subjuegos entre las decisiones de política monetaria y las decisiones de política fiscal en Colombia. Se constató para cada uno de los escenarios planteados en el ejercicio la existencia de equilibrios perfectos en subjuegos en el sentido de Nash, que permitieron develar que la condición de racionalidad maximizadora de los jugadores es el elemento principal a la hora de la toma de decisiones al interior de grupos sociales. Por último, la observación puso en evidencia que las interacciones entre dos agentes están condicionadas por las estrategias que adopte el agente que opere de líder, y que las decisiones del agente seguidor regularmente se basan en criterios cooperativos y de coordinación; en este sentido, se pudo establecer que el Banco de la República determina la manera en la que el Gobierno, respetando los conductos constitucionales, toma sus decisiones de política con el fin de mantener la estabilidad económica del país.

Palabras clave: Política Monetaria, Política Fiscal, Teoría de Juegos, Equilibrio de Nash, Economía Experimental.

Clasificación JEL: E62, E52, C70, C90.

1. Magíster en Ciencias Económicas Economía, docente tiempo completo de la Universidad Cooperativa de Colombia sede Santa Marta. Coordinador del Grupo de investigación UMBRALES y docente investigador adscrito al programa de Administración de Empresas. E-mail: raul.rodriguez@ campusucc.edu.co

2. Economista de la Universidad del Magdalena. Correo electrónico: j_economia@yahoo.es

3. Economista de la Universidad del Magdalena. Correo electrónico: e_romo04@yahoo.es

Fecha de recepción: 7 de enero de 2014 - Fecha de aprobación definitiva: 14 de marzo de 2014 


\title{
FISCAL AND MONETARY POLICY IN COLOMBIA: AN EXPERIMENTAL ANALYSIS FROM THE COORDINATED DECISION MAKING
}

\author{
By: Raúl Rodríguez Luna - Jairo Mendoza - Erik Romo Salas
}

\begin{abstract}
In this paper the results of a simulation are discussed from methods of experimental and modeling economy with elements of game theory, where a sequential game that allowed finding equilibria perfect Nash subgame between decisions were made monetary policy and fiscal policy decisions in Colombia. It was found for each of the scenarios presented in exercising the existence of subgame perfect equilibria in the sense of Nash, who helped unveil the condition of maximizing rationality of players is the main element in the decision making inside of social groups. Finally, the observation revealed that the interactions between two agents are conditioned by the strategies adopted by the agent that operates leader, and that the decisions of the follower agent regularly rely on cooperative approaches and coordination, in this sense, it was established that the Central Bank determines the manner in which the Government, respecting the constitutional channels, making its policy decisions in order to maintain economic stability.
\end{abstract}

Key words: Monetary Policy, Fiscal Policy, Game Theory, Equilibrio de Nash, Experimental Economics.

JEL Classification: E62, E52, C70, C90. 


\section{INTRODUCCIÓN}

Las decisiones de política económica afectan el comportamiento, en principio, de variables macroeconómicas reales tales como el crecimiento del producto, la oferta y la demanda agregada, la inversión, el ahorro, los precios, el empleo y los salarios, y de las cuentas del sector externo. En Colombia, la autoridad monetaria -El Banco de la República- tiene entre sus funciones alcanzar y lograr que el producto crezca alrededor de su tendencia de largo plazo. Por otra parte, las políticas fiscales son definidas desde los poderes ejecutivo y legislativo, considerando las líneas de trabajo que plantea cada gobierno ${ }^{4}$. De acuerdo con lo anterior, la coordinación entre la política monetaria y la política fiscal es fundamental para el adecuado desempeño de la economía, la principal razón es que en el corto plazo, los objetivos trazados por las autoridades pueden resultar incompatibles; también es importante debido a que las acciones de política fiscal pueden condicionar las decisiones de política monetaria y viceversa, poniendo en riesgo la estabilidad de la economía ante perturbaciones exógenas. Es decir, si las autoridades de política económica no asumen una posición coordinada entre sus acciones, el resultado potencial sería una fuerte inestabilidad de la economía, con altas tasas de interés, presiones del tipo de cambio, un acelerado aumento del nivel de precios y por supuesto, un impacto negativo sobre el crecimiento del producto.

Diversos trabajos han demostrado que la coordinación entre las autoridades de política macroeconómica es necesaria; sin embargo, no es un tema reciente. En particular, Woodford (1994) señaló que la política fiscal debe ser considerada como política dominante, en tanto que determina en términos nominales el nivel de precios en una economía con banco central independiente. De manera que la estabilidad de los precios se logra en la medida en que la autoridad monetaria asuma este objetivo como su bandera, y la autoridad fiscal asuma la disciplina de satisfacer su restricción presupuestaria intertemporal (Donayre y González, 2002).

Ahora bien, si la misión del Banco de la República es controlar la inflación, y para ello hace uso de "instrumentos" de política, que a su vez pueden transmitir efectos sobre algunas de las variables reales descritas antes, como por ejemplo el empleo ¿cómo debe actuar el gobierno ante esta situación? si, con razón, ningún gobierno quiere altas tasas de desempleo.

En este sentido, las decisiones de gasto fiscal tomadas por el gobierno se ven limitadas por las consecuencias que puedan derivar en el nivel de precios, y a su vez los decisores de la política monetaria manipulan con cautela instrumentos como las tasas de interés y las $\mathrm{OMAs}^{\mathbf{5}}$, conscientes de sus potenciales efectos si no se toma la decisión adecuada.

Dado este panorama, ¿Es posible establecer con certeza las mejores decisiones de política, tanto las monetarias como las fiscales?, ¿Permite el contexto colombiano encontrar equilibrios de Nash perfectos en subjuegos a partir de escenarios

4. En este trabajo para mayor simplicidad se considerarán como objetivo principal del Banco el control de la inflación, y por parte del Gobierno el crecimiento del producto.

5. Operaciones de Mercado Abierto. 
simulados para la toma de decisiones de política económica? ¿Existe coordinación entre las autoridades de política económica, dados los escenarios planteados?

Para dar respuesta a las preguntas formuladas arriba, se diseñó un juego secuencial $^{6}$ de política monetaria y política fiscal haciendo uso de métodos de la economía experimental ${ }^{7}$, a través del cual se pudo establecer los equilibrios de Nash perfectos en subjuegos correspondientes a cada uno de los escenarios planteados, en el marco de los objetivos trazados por las autoridades de política económica en Colombia. Dicho juego permitió además hallar la solución de cada juego-escenario por inducción hacia atrás, y encontrar las correspondencias de mejor respuesta para cada escenario.

Frente a lo expuesto, este documento se divide en seis secciones incluida esta introducción. En la segunda se hace una revisión de la literatura que aborda los temas de coordinación de política económica. En la tercera, se presenta el marco teórico que guía el trabajo. En la cuarta sección se exponen los aspectos metodológicos de la investigación mientras que en la quinta se enseñan los resultados obtenidos a partir del ejercicio de simulación. Finalmente en la sexta sección se esbozan las conclusiones del trabajo.

\section{REVISIÓN DE LA LITERATURA}

La interacción entre la política fiscal y la política monetaria representa hoy por hoy una cuestión de primera página en las agendas internas de muchos países. El tema fue abordado formalmente por Sargent y Wallace (1981) quienes afirman que bajo un régimen en el que la política fiscal es dominante, el control de la autoridad monetaria sobre la inflación es muy limitado aun cuando la base monetaria y el nivel de precios se mantengan estrechamente vinculados, y sobre todo cuando la tasa de interés supera la tasa de crecimiento del producto; demostraron también que lo anterior es cierto cuando además las políticas monetaria y fiscal son ejecutadas coordinadamente. Por su parte las decisiones de política muchas veces se toman con base en las políticas monetarias futuras.

En este sentido, se revelaría que un equilibrio de expectativas racionales se da siempre y cuando cumpla con dos características. En primer lugar, las reglas de decisión para los agentes privados que determinan sus acciones es óptima dadas sus expectativas estimadas a partir de una función, que a su vez determina las expectativas de los agentes como una función de información actual. En segundo lugar, el equilibrio es óptimo para el formulador de políticas, el cual sigue sus propias reglas que especifican el comportamiento de los instrumentos de política

6. Un juego secuencial es un juego donde existe un orden de juego, y cuando todos los jugadores han jugado cada uno recibe su ganancia. Después de distribuir las ganancias, el juego está acabado (Haeringer, 2004). En un juego secuencial, los jugadores poseen información completa y perfecta, y esta es la principal razón por la que en este trabajo se optó por seguir este tipo de juegos, ya que el segundo jugador conocía de antemano las decisiones tomadas por el primero, y las posibles consecuencias que esas decisiones comportarían.

7. Se diseñó un juego en el que se simularon las sesiones de la Junta Directiva del Banco de la República (Autoridad Monetaria) y del Consejo de Política Fiscal (Confis) del Ministerio de Hacienda y Crédito Público del Gobierno Nacional (Autoridad Fiscal). 
como función de un conjunto de información, lo que le permite actuar de acuerdo con las expectativas de los agentes privados, ya que el formulador conoce la forma de las reglas de decisión de los agentes.

Es decir, el formulador de políticas intenta maximizar un objetivo que refleja las preferencias de la "sociedad" respecto a la inflación y el desempleo.

A partir de esto, la política de equilibrio planteada por Barro y Gordon (1983) establece que:

$$
\hat{\pi}_{t}=\frac{a \alpha}{b}(1-k) E_{t-1} U_{t}^{\prime \prime}=\pi_{t}^{e}
$$

$$
\text { Si } \hat{\pi}_{t}=\pi_{t}^{e} \text {, entonces } U_{t}=U_{t}^{\prime \prime} \text { también constituye el equilibrio }
$$

Donde $\hat{\pi}_{t}$ corresponde a la tasa de inflación y $\pi_{t}^{e}$ la tasa de inflación esperada; mientras que $U_{t}$ representa la tasa de desempleo $U_{t}$ y $U_{t}^{\prime \prime}$ la tasa natural de desempleo.

La anterior ecuación, que determina la política de equilibrio, provee un equilibrio de Nash debido a que, dada la percepción que los agentes $\left[\pi_{t}^{e}=h^{e}\right]$ tienen de la política, la minimización de los costos $\left(E_{t-1}\right)$ que esta genera induce al formulador de políticas a establecer una meta de inflación para cada período $\left[\hat{\pi}_{t}=h^{e}\right]$, en tanto que, si las expectativas de los agentes son racionales, estos optimizan sujetos a tales expectativas.

Las decisiones estratégicas de coordinación de políticas monetarias y fiscales son escasamente modeladas en Colombia, de manera que los principales trabajos dan cuenta de análisis basados en el comportamiento de las variables macroeconómicas, más que en los procesos decisores que conducen a la ejecución de instrumentos de política.

Al respecto, algunos autores han analizado las relaciones dinámicas entre el Gobierno y el Banco Central (Vargas, 1994) y entre el crecimiento económico y la inflación (Benavides y Cárdenas, 2005). El primero concentró su estudio en la revisión de las valoraciones que los agentes -Gobierno (G), Banco Central (BC) y Sector Privado (SP)- asignan a sus objetivos, presentando algunos ejemplos derivados a partir de un modelo estático de oferta y demanda agregadas en una economía abierta, con expectativas racionales. Los resultados obtenidos le permitieron al autor afirmar que la inflación y la tasa de cambio real de equilibrio están determinadas por la distribución de la información entre el BC, el G y el SP; así como también presentar indicios sobre las condiciones en las cuales el G y el BC encuentran provechoso actuar de manera coordinada. Por último, concluyó que para mantener una tasa de inflación baja y estable el Banco Central debe conservar su independencia, siendo condición sine qua non que el Gobierno no se encuentre en una posición inicial ventajosa frente al Banco. 


\section{MARCO TEÓRICO}

Imaginemos un mundo, coloquemos en él a dos personas ${ }^{8}$ que se encuentran en una situación hipotética, estas personas deben elegir entre hacer una cosa y hacer otra; las decisiones que tomen les servirán para mejorar su bienestar o para empeorarlo, de modo que uno de estos jugadores debe elegir bien sus estrategias y esperar que sea la adecuada frente a las estrategias que seguirá el otro jugador ${ }^{9}$, para obtener una buena recompensa.

El análisis de este tipo de situaciones constituye la "Teoría de Juegos”, cuyas más célebres aplicaciones teóricas fueron expuestas por John Von Neumann y Oskar Morgenstern en 1944, en su libro: Theory of Games and Economic Behavior. La propuesta original de los autores consistió en introducir en su análisis las decisiones que toma la gente en sus hogares, en la calle, en las empresas, etc., teniendo en cuenta que no siempre se posee el control total sobre las variables que inciden en el resultado final; sin embargo, este tipo de interacciones fueron planteadas y desarrolladas inicialmente desde los trabajo de Augustin Cournot (1838) y de Joseph Louis François Bertrand (1883), se abre una agenda de discusión muy interesante de la teoría económica, relacionada con el papel de las interacciones entre firmas al momento de reconocer diferentes estructuras de mercado los esfuerzos de modelación se hicieron progresivamente más visibles durante los albores del siglo XX, cuando surgen trabajos como el de Chamberlin (1933) y Stackelberg (1934), quienes refuerzan esta tendencia de modelación. Trabajos como los de Shapiro (1996), Werden (1997), Epstein y Rubinfeld (2001), relacionados con simulación de integraciones empresariales dan cuenta de esta tendencia.

En esta dirección las decisiones de política fiscal y política monetaria son igualmente tomadas de forma estratégica por las autoridades responsables de estas, haciendo uso de los mecanismos que constitucionalmente se les ha provisto y que les permiten alcanzar sus objetivos individuales. No obstante, las autoridades monetaria y fiscal ejecutan sus acciones teniendo en cuenta las potenciales consecuencias sobre las acciones de una sobre la otra y obviamente sobre sus objetivos.

Dicho juego (que implica las decisiones de política fiscal tomadas por el Gobierno Nacional frente a las decisiones de política monetaria adoptadas por el Banco de la República) al ponerse en marcha, adopta características de un juego $G$ compuesto por $(N, S, u)$, donde $N=(1,2, \ldots, n)$ y representa el conjunto de jugadores -en este caso sólo el Gobierno Nacional y el Banco de la República- mientras que $S=\Pi_{i=1}^{n}$ corresponde al conjunto de estrategias de que disponen el conjunto de jugadores y particularmente $S_{\boldsymbol{i}}$ es el conjunto de estrategias con las que cuenta el jugador $i$; por último, $u=\left(u_{i}, \ldots, u_{n}\right)$ es la función de pagos o recompensas que obtendrán los jugadores durante o al finalizar el juego.

En este sentido, las interacciones dadas en política económica en Colombia, surgen de un escenario en el que los agentes involucrados poseen información

8. En adelante también se les llamará agentes o jugadores.

9. En este ejemplo los jugadores saben con certeza como actuará cada uno (supuesto de información simétrica). 
completa y perfecta ${ }^{\mathbf{1 0}}$, pues las funciones de pago son de dominio público, además, el comportamiento que asumen es completamente dinámico, dado que disponen de varias acciones como estrategias de juego para adoptar en el tiempo. Esto supone que el jugador -el Banco de la República, por ejemplo- al que le corresponde decidir en un momento determinado conoce la historia de las decisiones que fueron tomadas previamente por él mismo o por el Gobierno, esta misma condición es igualmente válida en el sentido contrario.

Entonces, considerando el escenario descrito, supongamos la existencia de un juego con dos jugadores, el Gobierno Nacional y el Banco de la República. Cada jugador dispone de un determinado número de acciones como estrategias de juego $a_{i=1}^{n} \in S_{i=1}^{n}$. Así pues, un perfil de estrategias $\boldsymbol{s}$ es una lista de estrategias $\left(s_{1}, s_{2}, \ldots, s_{n}\right)$ una para cada jugador. De forma que, dado cualquier perfil de estrategias $\boldsymbol{s}$, cada uno de los jugadores obtiene un resultado $\boldsymbol{\mu}_{i}$, el cual puede depender de todo el conjunto de acciones que componen el perfil.

Como el objetivo del trabajo es hallar los equilibrios de Nash perfectos en subjuegos dados los escenarios simulados de política fiscal y política monetaria, lo anterior permite plantear un modelo de juego secuencial ${ }^{11}$ en el que existe información completa y perfecta. En este sentido la autoridad monetaria establece un conjunto de acciones así:

$$
P M=\left(P M^{E}, P M^{C}\right)
$$

Donde cada acción corresponde a:

$$
\begin{aligned}
& P M^{E} \text { : Realizar Política Monetaria Expansiva } \\
& P M^{C} \text { : Realizar Política Monetaria Contractiva }
\end{aligned}
$$

Dentro de este conjunto de estrategias ${ }^{12}$ la autoridad monetaria puede realizar sus acciónes por otra parte el jugador Gobierno Nacional dispone del siguiente conjunto de estrategias:

$$
P F=\left(P F^{E}, P F^{C}\right)
$$

Donde cada estrategia corresponde a:

10. Esto es un supuesto aunque es muy fuerte se hace con motivos de simplificación. Este tipo de interacciones presentan claramente información asimétrica.

11. Un juego secuencial permite que uno de los jugadores tome una decisión primero y ejecute una acción de un conjunto de acciones que posee como estrategias; acto seguido el segundo jugador ejecuta una acción tomada de un conjunto de acciones de que dispone como estrategias. Los pagos o recompensas obtenidos por cada jugador dependerán de las acciones que fueron tomadas y del número de etapas que duró el juego.

12. Las estrategias generales que tiene el Banco podemos encontrar un conjunto de acciones que le permiten llevar a cabalidad estas estrategias, tales acciones son aumentar las tasas de interés, realizar OMAs (Comprar y/o vender títulos, bonos, etc.), acumular reservas, incrementar el encaje bancario, bajar las tasas de interés, no realizar OMAs, desacumular reservas, disminuir el encaje bancario. 


\section{$P F^{E}$ : Política Fiscal Expansiva \\ $P F^{C}$ : Política Fiscal Contractiva}

De igual manera que el Banco, el Gobierno tiene un conjunto de acciones ${ }^{13}$ que le permiten ejecutar sus políticas o estrategias.

En cuanto a las utilidades, su estimación surge de los beneficios que se obtienen dada la combinación de acciones tomadas por cada jugador. De tal forma que las recompensas para cada jugador se determinan según los escenarios de cada combinación de acciones:

Utilidades para el Banco de la República: $U_{B}\left[\left(\pi-\pi^{*}\right),\left(y-y^{*}\right),(P M)\right]=B E_{B}^{m f}$

Utilidades para el Gobierno Nacional: $\quad U_{G}\left[\left(y-y^{*}\right),\left(\pi-\pi^{*}\right),(P F)\right]=B E_{G}^{f m}$

Lo anterior se puede representar en un árbol de decisiones según cada estrategia:

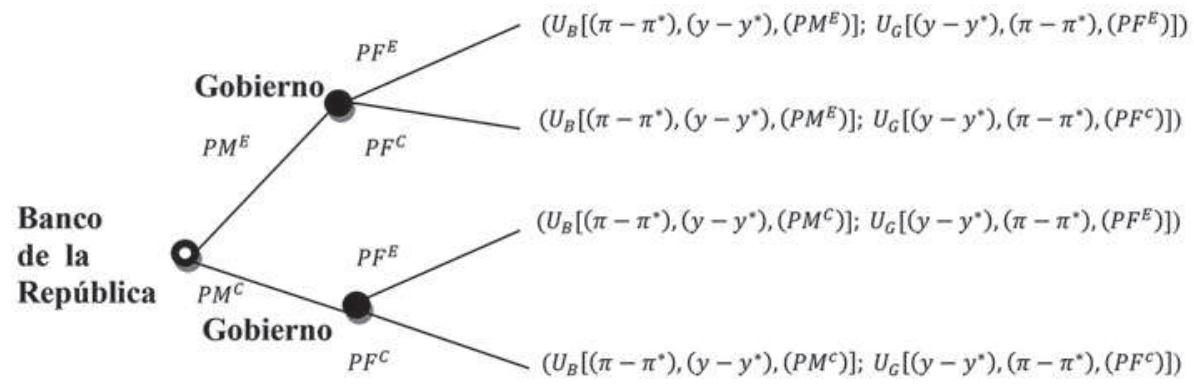

El método a seguir para establecer los equilibrios de Nash perfectos en subjuegos es el de inducción hacia atrás ${ }^{\mathbf{1 4}}$, este supone que el jugador 2 , en este caso el Gobierno Nacional, dispone de un problema de maximización del siguiente tipo:

$\operatorname{Max} U_{G}\left[\left(y-y^{*}\right),\left(\pi-\pi^{*}\right),(P F)\right]$

Donde,

$$
\max _{P M \in S_{B}} U_{B}\left[\left(y-y^{*}\right),\left(\pi-\pi^{*}\right),(P F)\right], R_{G}\left(P M_{B}\right)
$$

13. Aumentar el gasto público, aumentar los impuestos, disminuir el gasto público, disminuir los impuestos.

14. Este procedimiento analiza el juego desde el final hacia el principio: desde los conjuntos de información en el final del árbol hasta los conjuntos de información del principio. Permite identificar el equilibrio de Nash perfecto en subjuegos. 
Así pues, el problema de maximización del Banco de la República tiene también una solución y que no hay razones para pensar que esta sea única $P M^{*}$. Por lo tanto $\left[P M^{*}, R_{G}\left(P M^{*}\right)\right]$ es el resultado por inducción hacia atrás del juego.

Para este caso se referencia el trabajo de Stackelberg, cuando plantea que, si en un mercado con un bien y dos organizaciones en el que está restringida la entrada a otras firmas. Cada firma trata de producir una cantidad teniendo en cuenta la producción de la otra organización a diferencia de Counot la empresa líder conoce la conducta de la firma seguidora, produciendo una determinada cantidad según sus intereses según la cual la firma seguidora deberá producir una cantidad distinta. Se da, por tanto, una clara ventaja de la empresa que realiza un movimiento con anticipación.

El trabajo de Stackelberg ha sido base de muchos de los juegos desarrollados por Nash. A partir del modelo de Stackelberg, Nash plantea diferentes alternativas en torno a una serie de jugadas interpretadas por dos duopolistas que controlan el mercado mediante esta serie de juegos se ha encontrado que no sólo adquiere ventaja el primero en mover, sino que bajo ciertas condiciones ser el primero puede resultar una desventaja decisiva toda vez que influye la capacidad decisiva de la innovación tecnológica.

En esta línea argumental, se podría inferir que el líder en este juego es el Banco; este argumento se basa además en el hecho de que la política adoptada por el Banco se aplica de manera más inmediata (por no decir que automática), que aquella ejecutada por el Gobierno -el cual actúa como la firma seguidora- ya que la política fiscal para ser aplicada requiere en ocasiones de trámite legislativo, voluntad política, existencia de recursos, entre otros, lo cual hace que pierda inmediatez en momentos determinados, en contraste con las decisiones tomadas por el Banco que no tiene que realizar estos trámites dada la autonomía e independencia de que goza por mandato constitucional ${ }^{15}$.

En otras palabras, las autoridades de política buscan maximizar una función de utilidad; de acuerdo con esto, un nivel de utilidad para la autoridad monetaria se denota como $U_{\boldsymbol{B}}$ y sus objetivos están asociados a los coeficientes $\alpha^{B}, \beta^{B}$, $\delta$, que miden de manera respectiva el costo en que se incurre con la caída del producto respecto a su tendencia, un aumento de la inflación sobre el nivel esperado, y a las desviaciones de los efectos positivos de las políticas monetarias ejecutadas, por lo cual $\alpha^{B}, \beta^{B}, \delta \geq 0$. De forma que el nivel de utilidad del Banco de la República se representa por:

$$
\begin{aligned}
& U^{B}=f\left[\left(\pi-\pi^{*}\right),\left(y-y^{*}\right),(P M)\right] \\
& U^{B}=-\propto^{B}\left[\min \left(y-y^{*}, 0\right)\right]^{2}-\beta^{B}\left[\max \left(\pi-\pi^{*}, 0\right)\right]^{2}-\delta(P M)^{2}
\end{aligned}
$$

15. Para simplificar el análisis, se soslayan del escenario las variables corrupción y guerra interna, que pueden incidir en las decisiones tomadas por cada uno de los jugadores, y se asume un estado en que los decisores de política actúan buscando constantemente el mayor bienestar de la población. 
La función de utilidad del Gobierno comporta la misma estructura; los coeficientes $\alpha^{G}, \beta^{G}$ y $\tau$ miden respectivamente la caída del producto respecto a su tendencia, un aumento de la inflación sobre el nivel esperado, y a las desviaciones de los efectos positivos de las políticas fiscales ejecutadas:

$$
\begin{aligned}
& U^{F}=f\left[\left(\pi-\pi^{*}\right),\left(y-y^{*}\right),(P F)\right] \\
& U^{F}=-\propto^{F}\left[\min \left(\pi-\pi^{*}, 0\right)\right]^{2}-\beta^{F}\left[\max \left(y-y^{*}, 0\right)\right]^{2}-\delta(P F)^{2}
\end{aligned}
$$

Frente a lo anterior, los niveles perseguidos por las autoridades para sus variables de interés -siguiendo el modelo de De Gregorio (1996)- quedan expresados de la siguiente forma:

$$
\begin{aligned}
& \pi-\pi^{*}=\lambda_{y}\left(y-y^{*}\right)-\lambda_{0} \\
& y-y^{*}=\gamma_{F}(P F)-\gamma_{M}(P M)+\gamma_{0}
\end{aligned}
$$

Donde $\pi-\pi^{*}$ constituye la desviación del nivel de inflación frente a su tasa óptima, mientras que $y-y^{*}$ representa la brecha del producto. Por su parte $\gamma_{F}$ y $\gamma_{M}$ representan respectivamente la elasticidad de la brecha del producto con respecto al instrumento de la política fiscal y al instrumento de la política monetaria, y $\lambda_{y}$ la elasticidad de la inflación frente a la brecha del producto. Finalmente, $\gamma_{0}$ y $\lambda_{0}$ representan de manera respectiva los choques exógenos de demanda y oferta agregadas. Las ecuaciones (11) y (12) comportan correspondientemente funciones de demanda y oferta agregadas.

Un shock positivo de la oferta, es decir $\lambda_{0}>0$, no merece mayor análisis en este trabajo, basta decir que produce un nivel de inflación más bajo que $\pi^{*}$ y un producto más alto que $y^{*}$, razón por la cual las autoridades no tienen incentivos para intervenir a través de los diversos instrumentos de política de que disponen. Sin embargo, ante un shock negativo de oferta la producción disminuye y la inflación aumenta, induciendo a una reacción de política por parte de las autoridades ante una posible pérdida.

De acuerdo con esto, las funciones de pérdida para el Banco de la República y el Gobierno pueden escribirse de la siguiente forma:

$$
\begin{aligned}
& U^{B}=\alpha^{B}\left[y f(P M, P F)-y^{*}\right]-\beta^{B}\left[\pi f(P M, P F)-\pi^{*}\right]+\delta(P M) \\
& U^{G}=\alpha^{G}\left[y f(P M, P F)-y^{*}\right]-\beta^{B}\left[\pi f(P M, P F)-\pi^{*}\right]+\tau(P F)
\end{aligned}
$$

Si el Banco de la República hace uso de un instrumento de política como primer jugador, seguido del Gobierno como segundo jugador, las condiciones de primer orden para la maximización están dadas por

$$
\begin{aligned}
& \frac{\partial U^{B}}{\partial P M}=\alpha^{B} y^{\prime}-\beta^{B} \pi^{\prime}+\delta=0 \\
& \frac{\partial U^{G}}{\partial P F}=\alpha^{G} y^{\prime}-\beta^{G} \pi^{\prime}+\tau=0
\end{aligned}
$$


Obsérvese que si el Banco de la República hace uso de un instrumento de política como primer jugador, seguido del Gobierno como segundo jugador, se obtiene un equilibrio determinado por los costos en que se incurre con la caída del producto respecto a su tendencia, y un aumento de la inflación sobre el nivel esperado de los precios con la desviaciones de los efectos de las políticas monetarias ejecutadas.

\section{ASPECTOS METODOLÓGICOS}

El ejercicio experimental se llevó a cabo en un salón en el cual se simularon sesiones de la Junta Directiva del Banco de la República y del Consejo de Política Fiscal (Confis) del Gobierno. Tanto la junta del Banco como la junta del Confis estuvieron integradas por profesores de la Facultad de Ciencias Empresariales y Económicas y destacados estudiantes y egresados del Programa de Economía de la Universidad del Magdalena, cuyos perfiles y conocimientos constituyeron el principal criterio de selección. Antes de iniciar las sesiones, los participantes fueron instruidos en la mecánica del ejercicio; el moderador describió el objeto de la investigación y procedió a explicar la metodología.

Los participantes fueron agrupados en cada una de las juntas (las cuales configuraban los jugadores) de acuerdo con sus perfiles y conocimientos. Se les explicó que el ejercicio consistiría en el planteamiento de cuatro escenarios sobre los cuales debían tomar decisiones de política económica, primero de manera individual y autónoma y posteriormente, de manera conjunta, de modo que estas últimas representaran las posiciones de cada jugador que representaban, es decir, del Banco de la República y del Gobierno. El moderador señaló además que al finalizar el ejercicio se evaluarían, de acuerdo a criterios teóricos, las mejores decisiones individuales y se recompensaría a quienes tomaran las decisiones más acertadas con alguno de los siguientes dos artículos: un libro o un almuerzo. Estos artículos tenían el propósito de incentivar a los participantes a que decidieran de la mejor manera frente a cada uno de los escenarios planteados.

Previamente se estableció que los participantes que simulaban la Junta Directiva del Banco de la República debían sesionar primero, y posteriormente lo harían los que simulaban la junta de Confis, en consecuencia con los preceptos teóricos y prácticos de los juegos secuenciales. Antes de iniciar las sesiones, el moderador planteó a los participantes los supuestos que delimitan el juego propuesto en este trabajo:

1. Colombia es una pequeña economía abierta que permite libre entrada y salida de capitales y posee tipo de cambio flexible de flotación sucia ${ }^{\mathbf{1 6}}$. En esta economía la divisa de referencia es el dólar de Estados Unidos, el cual es el principal socio comercial del país. Adicionalmente, los precios son flexibles, por lo que además las variaciones en el nivel de precios internacional tiene implicaciones en los precios internos. Es por esta razón que las autoridades de política económica

16. Un tipo de cambio cuya fijación, además de la oferta y demanda de divisas, se determina de acuerdo a las intervenciones que el Banco Central realice en función de evitar variaciones bruscas en la cotización de la moneda nacional. 
han optado por fijar una meta anual de inflación ${ }^{17}$ que les permita cumplir con su mandato.

2. La política fiscal de Colombia es ejecutada por el Gobierno Nacional, con previa aprobación del Congreso de la República. Esto es, las decisiones que se tomen en materia de gasto público (Aumento o disminución), o carga tributaria ( $\mathrm{Au}-$ mento o disminución) deben ser avaladas en primera instancia por el Senado y la Cámara de Representantes.

3. De acuerdo con el supuesto anterior, es preciso aclarar que el Gobierno cuenta con dos principales estrategias de política fiscal. Una es la política fiscal expansiva (Aumento del gasto público o disminución de los impuestos) y la otra es la política fiscal contractiva (Disminución del gasto público o aumento de los impuestos).

4. El Banco de la República es la autoridad monetaria en Colombia; cuenta con siete miembros, de los cuales sólo el Ministro de Hacienda y Crédito Público no tiene voto en las decisiones. El Banco tiene como funciones constitucionales mantener una tasa de inflación baja y estable, conservar la capacidad adquisitiva de la moneda local y lograr que el producto crezca alrededor de su tendencia de largo plazo, entre otros objetivos. Para cumplir con su misión el Banco cuenta con una serie de instrumentos de intervención a saber: Tasas de interés, Operaciones de Mercado Abierto, Encaje Bancario y Reservas Internacionales. La manipulación de tales instrumentos le permite responder a las coyunturas económicas.

5. Para simplicidad del análisis propuesto, el jugador-público no participa en el juego y sus decisiones se consideran neutrales o dadas (ceteris paribus).

6. Tanto el Banco de la República como el Gobierno hacen sus proyecciones antes de entrar en sus sesiones, lo que les permite saber cuáles son las decisiones más convenientes para cada uno.

7. Los jugadores toman sus decisiones con base en las suposiciones de las jugadas de cada uno, usando la información de que disponen y de acuerdo con el contexto económico del momento.

8. En concordancia con los dos supuestos anteriores, ambos jugadores conocen las preferencias del otro.

9. Los jugadores pueden modificar sus preferencias de acuerdo a los escenarios económico, social y político presentes tanto nacional como internacionalmente.

10. Se supone racionalidad y criterios de cooperación y coordinación en la toma de decisiones del Banco de la República y del Gobierno.

11. El Banco de la República cuenta con ventaja frente al Gobierno, ya que prevé el posible comportamiento de éste en el futuro, ya que dada la información que

17. A esta propuesta se le conoce en la teoría económica como Esquema de Inflación Objetivo, bajo el cual el Banco de la República fija todos los años una meta de inflación para anclar las expectativas de los agentes privados. 
posee infiere la política fiscal y en este sentido sus decisiones condicionan las del Gobierno.

12. Tanto el Banco de la República como el Gobierno toman sus decisiones de tal suerte que puedan alcanzar sus objetivos particulares y en consecuencia, mejorar el bienestar de la población.

\subsection{El ejercicio de simulación}

Este ejercicio experimental sigue los aportes de Davis y Holt (1992) citado en Gutiérrez, Guzmán y Jiménez (2000: 120) el cual intenta proponer la realización de trabajos que reproduzcan y validen los resultados obtenidos en este experimento.

De acuerdo con Smith (1994) existen por lo menos siete razones importantes en la literatura por la que los economistas pueden hacer uso de los experimentos, a saber:

1. Probar una teoría

2. Explorar las causas del fracaso de una teoría

3. Establecer regularidades empíricas como base para una nueva teoría

4. Comparar los entornos

5. Comparar instituciones

6. Evaluar las propuestas de política

7. Establecer un laboratorio de campo de pruebas para el diseño de instituciones.

El ejercicio se dividió en dos etapas: en la primera se desarrolló una sesión simulada de la Junta Directiva del Banco de la República (Agente Líder), y en la segunda una sesión del Consejo de Política Fiscal -Confis- del Ministerio de Hacienda y Crédito Público (Agente Seguidor).

\subsection{Estructura del ejercicio de simulación}

Se consideró un juego de dos jugadores en el que un primero (Banco de la República - BR), interactúa secuencialmente con otro (Gobierno Nacional - G). El juego está planteado para que las interacciones se realicen en cuatro períodos, correspondientes a los cuatro trimestres de un año. Las decisiones de cada jugador fueron tomadas siguiendo un modelo de juego secuencial, teniendo en cuenta que se trata de escenarios en los que el segundo jugador posee información completa y perfecta sobre las decisiones que tome el primer jugador. Mientras que el primero prevé los posibles movimientos del segundo y en consecuencia actúa.

\section{En el primer momento se propone el siguiente escenario:}

En los últimos años ${ }^{18}$ el peso se ha apreciado respecto al dólar; en el 2003 se situaba alrededor de 3.000 pesos, en contraste con lo anterior, en los últimos

18. Para poner en contexto el experimento se desarrolló con un grupo de estudiantes universitarios durante 2010-2013. 
meses se ha ubicado cercano a los 1.900 pesos; de continuar con esa tendencia se generarían efectos nocivos para los exportadores. Se estima que en los últimos 10 años los exportadores han despedido más de 15.000 empleados a causa de los desajustes cambiarios, afectando la economía doméstica; en caso de que el Banco de la República compre dólares ¿Qué acción adicional debería adoptar para evitar que se ponga en riesgo el cumplimiento de la meta de inflación en el largo plazo?
a. Aumentar las tasas de interés
b. Disminuir las tasas de interés
c. Acumular reservas internacionales
d. Desacumular reservas internacionales
e. Aumentar el encaje bancario
f. Disminuir el encaje bancario

En caso de que el Banco decida comprar dólares y buscara ejecutar una acción complementaria ¿Cuál sería la respuesta del Gobierno si se encuentra en una situación fiscal deficitaria y conoce la decisión tomada por el Banco frente a la anterior situación?
a. Aumentar el gasto público
b. Aumentar los impuestos
c. Disminuir el gasto público
d. Disminuir los impuestos

\section{Posteriormente se plantea un segundo momento del ejercicio, en el que el escenario tiene las siguientes características ${ }^{19}$ :}

En lo corrido del año 2013 la inflación ha registrado un bajo nivel (-0,26\% en el mes de mayo). Adicionalmente, los indicadores de inflación básica (los que excluyen los precios de los productos más volátiles, como los alimentos) continúan a la baja. De otra parte, las expectativas de inflación igualmente disminuyeron y se sitúan en el rango meta fijado por la Junta Directiva del Banco de la República para el 2013, que coincide con el de largo plazo (entre $2 \%$ y 4\%). Como quiera que el comportamiento a la baja de la inflación ha estado jalonado por los precios de los alimentos y de los regulados, cabe destacar que se observa una reducción relativamente generalizada de precios, abarcando en consecuencia una amplia variedad de bienes y servicios. Por lo anterior, la inflación a fin del año se estima que se ubicará por debajo del punto medio del rango meta de largo plazo (3\%).

Por otro lado, el contexto externo ha mejorado en los últimos meses, lo que ha provocado que los analistas internacionales mejoren sus pronósticos de crecimiento económico para el 2014 en la mayoría de los países, y en particular en los Estados Unidos. Sin embargo, las proyecciones de crecimiento para Venezuela prevén una caída importante del PIB para el mismo año.

19. Basado en el artículo "Banco de la República reduce 50 puntos básicos su tasa de interés de intervención” publicado en http://www.desarrolloeconomico.gov.co/index.php/Historial/banco-de-larepublica-reduce-50-puntos-basicos-su-tasa-de-interes-de-intervencion.html. 
En busca de afianzar la recuperación de la economía nacional y reducir los efectos negativos de la caída del comercio con Venezuela como consecuencia de los conflictos diplomáticos entre los países, y sumando el hecho de que en Colombia hay elecciones presidenciales en 2014 y un posible cambio de gobierno hacia uno de extrema derecha.

¿Cuál sería la mejor acción a seguir por el Banco?

a. Aumentar las tasas de interés

b. Disminuir las tasas de interés

c, Acumular reservas internacionales

d. Desacumular reservas internacionales

e. Aumentar el encaje bancario

f. Disminuir el encaje bancario

g. Realizar operaciones de mercado abierto

h. No realizar operaciones de mercado abierto

¿Cuál sería la mejor acción a seguir por el Gobierno?

a. Aumentar el gasto público (conceder subsidios)

b. Aumentar los impuestos

c. Disminuir el gasto público

d. Disminuir los impuestos

\section{En el tercer momento se propone el siguiente escenario ${ }^{20}$ :}

El 2013 empezó con un dólar que se negociaba aproximadamente a 1.700 pesos. Ayer, en el mercado interbancario, la moneda norteamericana cerró cercano a 1.900 pesos, es decir, a uno 200 pesos por debajo frente a comienzo de año.

Para los exportadores, un dólar barato es grave, pues merma sus ingresos. Algunas industrias, como calzado y confecciones, que tienen que competir con productos importados, se ven presionadas puesto que pierden competitividad frente a todo lo que llega a dólar más barato. Y mientras estos sufren, otros disfrutan de un dólar bajo: el comercio que vende productos importados como computadores, electrodomésticos y la industria automotriz, hacen su agosto. También ganan las empresas que traen materias primas y maquinaria para renovar su aparato industrial.

Por otra parte, al ciudadano del común le cae muy bien la apreciación porque puede adquirir productos a bajos precios y quienes tienen deudas en dólares deben destinar menos pesos para pagar sus obligaciones. Los viajeros al exterior por turismo, negocios o de estudio, se favorecen con un dólar barato. Y hasta el Gobierno, pues se alivia el pago de la deuda externa, ya que reducir la relación deuda/PIB hace más robusta a la economía y la pone en capacidad de responder a choques externos negativos, como cambios en la liquidez y tasas de interés internacionales;

20. Basado en el artículo "La caída del dólar resta poder de competencia” publicado en http://www. elcolombiano.com/BancoConocimiento/L/la_caida_del_dolar_resta_poder_de_competencia1/ la_caida_del_dolar_resta_poder_de_competencia1.asp 
esta relación es uno de los principales factores que toman en cuenta las agencias calificadoras de riesgo al evaluar la capacidad de recuperación de las economías ante choques. Además, la apreciación ayuda a mantener una baja inflación porque los precios de los bienes y servicios importados se abaratan.

Los efectos devastadores de la apreciación obedecen, en algunos casos, a la falta de competitividad internacional que impide exportar; en otros, el dólar barato ha facilitado la competencia desleal y el contrabando.

Uno de los sectores más afectados con la apreciación del peso es el de la cadena textil-confección que dice que sólo este año ha perdido ingresos por cerca de 30 millones de dólares. Por su parte, la industria del calzado reporta una disminución de 1.425 empleos y tiene la capacidad instalada operando sólo al 50 por ciento.

Ante el contexto anterior ¿Cuál sería la decisión más acertada del Banco?

a. Realizar operaciones de mercado abierto (comprar TES)

b. No realizar operaciones de mercado abierto

c. Aumentar las tasas de interés

d. Disminuir las tasas de interés

e. Acumular reservas internacionales

f. Desacumular reservas internacionales

g. Aumentar el encaje bancario

h. Disminuir el encaje bancario

¿Cuál sería la decisión más acertada del Gobierno?

a. Aumentar el gasto público (conceder subsidios)

b. Aumentar los impuestos

c. Disminuir el gasto público

d. Disminuir los impuestos

\section{Finalmente, se plantea un último escenario con las siguientes características ${ }^{21}$ :}

La semana pasada, el Gobierno anunció un conjunto de medidas orientadas a disminuir las presiones revaluacionistas como terminar las monetizaciones (dejando de emitir deuda pública en dólares) que se venían haciendo este año; reducir a cero los aranceles a las importaciones de materias primas con el fin de incentivar la demanda por dólares requeridos para pagar por estos bienes; y mantener en cuentas externas 500 millones de dólares de dividendos provenientes de Ecopetrol que se esperaban recibir este año. Ahora la atención se centra en las decisiones que

21. Basado en el artículo "Lucha por controlar el dólar” publicado en http://www.portafolio.com.co/ negocios/consultorio/la-lucha-por-controlar-el-dolar_6410794-3 
tome el Banco de la República este viernes, el Gobierno se mantendrá a la espera de dichas decisiones para actuar.

A pesar de que las medidas del Gobierno podrían suavizar parcialmente la apreciación cambiaria, sus efectos serán limitados pues las principales presiones provienen de otros factores aún más dominantes. El principal de ellos sigue siendo la debilidad del dólar a nivel global. El abultado estímulo fiscal del Gobierno de Estados Unidos tendrá un impacto negativo en el déficit fiscal de los próximos años en ese país. A su vez, la política monetaria 'ultraexpansiva' de la Reserva Federal ha inundado el sistema global de dólares y ha generado incertidumbre con respecto a la inflación de largo plazo en Estados Unidos.

Estos dos factores han aumentado las primas de riesgo en los bonos del Tesoro de Estados Unidos, lo cual se transmite a otros activos positivamente correlacionados con el riesgo de ese país. En la medida que estas primas de riesgo han aumentado, los inversionistas globales han salido de este tipo de activos en busca de otras oportunidades de inversión. Esta situación ha implicado venta de dólares y menor demanda por dicha moneda. Simultáneamente, la aversión al riesgo global y las primas de riesgo de países emergentes, entre ellos Colombia, han disminuido significativamente en los últimos seis meses a niveles observados previos a la crisis.

En consecuencia, los inversionistas externos han dirigido sus flujos de inversión a activos de estos países (incluidas sus monedas). Además, las mejores expectativas de recuperación económica global, han tenido un efecto positivo sobre los precios de las materias primas durante el mismo período.

Este comportamiento es especialmente beneficioso para países exportadores de este tipo de bienes, como Colombia, pues tiene el potencial de acelerar su recuperación mientras aumenta el flujo de divisas a esos países.

Dicho esto, los factores anteriormente mencionados seguirán presentes en los meses por venir. Por eso, si el Banco de la República decide comprar dólares, los efectos alcistas que se podrían presentar sobre la tasa de cambio serán de carácter temporal.

Entonces, ¿Qué medidas complementarias deberá tomar el Banco?

a. Comprar TES y acumular reservas

b. Comprar TES y disminuir el encaje bancario

c. Aumentar las tasas de interés y vender TES

d. Aumentar las tasas de interés, desacumular reservas y aumentar el encaje bancario

e. Acumular reservas, comprar TES y disminuir el encaje bancario

f. Desacumular reservas y aumentar las tasas de interés

g. Aumentar el encaje bancario y vender TES

h. Disminuir el encaje bancario y disminuir las tasas de interés 
Dadas las medidas adoptadas por el Banco ¿Cuál seguirá siendo la posición del Gobierno?

a. Aumentar el gasto público (aumentar la emisión de deuda en moneda extranjera)

b. Aumentar los impuestos

c. Disminuir el gasto público (aumentar la emisión de deuda en moneda extranjera)

d. Disminuir los impuestos

\section{RESULTADOS}

A partir de los resultados obtenidos se extraen una serie de consideraciones empíricas, que más que cerrar la discusión sobre la importancia que pueda tener la política monetaria sobre la política fiscal y viceversa, amplían el espectro de análisis contribuyendo a la generación de nuevas hipótesis y al debate antiquísimo sobre la verdadera motivación de los agentes.

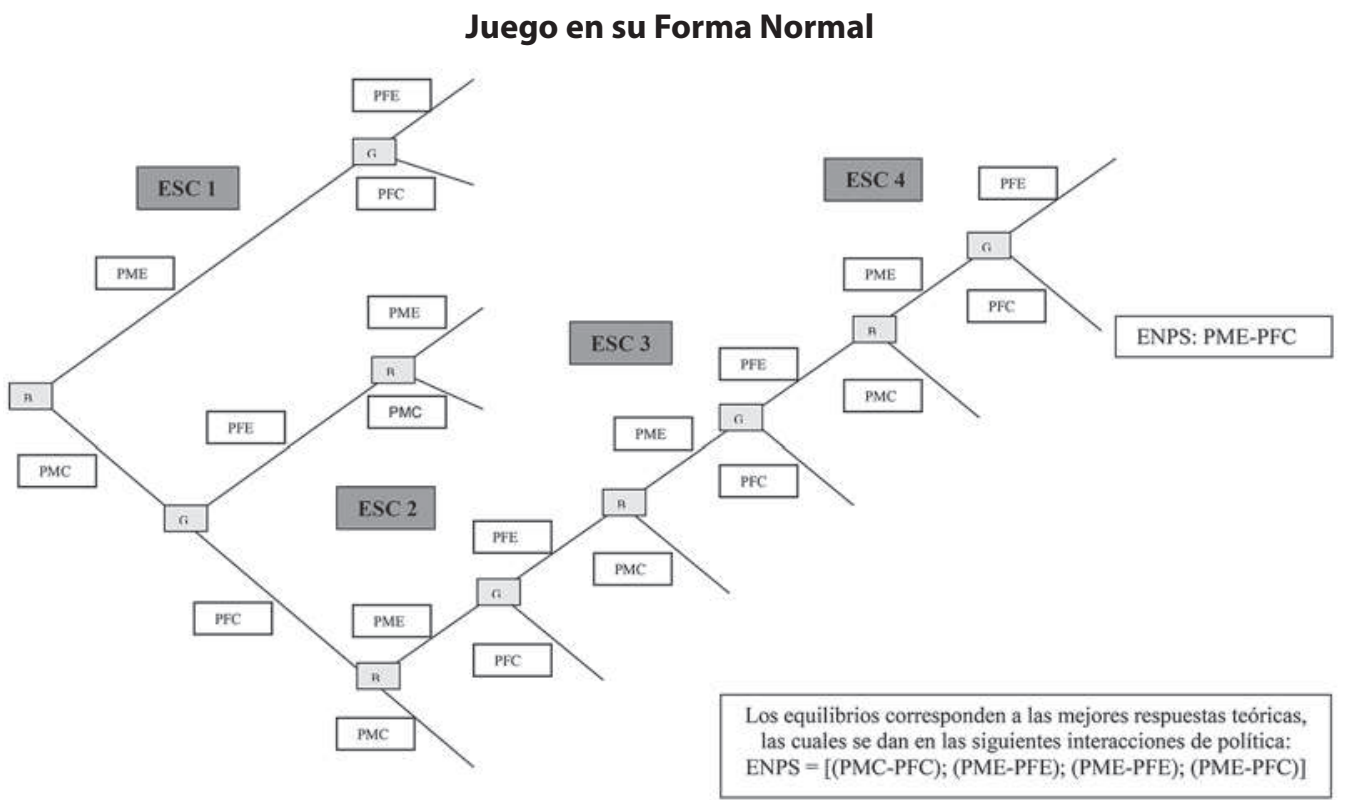

El análisis de los resultados permitió establecer los equilibrios de Nash perfectos en subjuegos para cada uno de los escenarios propuestos en las sesiones experimentales, y determinar las motivaciones de las autoridades para interactuar estratégicamente con criterios maximizadores.

Escenario 1. En concordancia con el escenario propuesto, se logró establecer que el Equilibrio se encuentra cuando el BR decide implementar una política monetaria contractiva mediante un incremento del encaje bancario, mientras que el G asume de igual manera una política fiscal contractiva aumentando los impuestos. 
Por otro lado, los agentes que intervinieron en el ejercicio por parte del Banco de la República tomaron la mejor decisión en términos de los beneficios que esta les reportaba, de manera que determinaron que la política acertada correspondía a una contracción monetaria implementada mediante un incremento en el encaje bancario, ante lo cual, el Gobierno asesorado por el Confis decidió adoptar una política fiscal expansiva incrementando el gasto público ¿Por qué el G tomó esta decisión? El argumento expuesto por la junta del Confis se basa en dar a la coyuntura propuesta un tratamiento de política fiscal contracíclica, orientada a reactivar algunos sectores de la economía, pero en particular al sector exportador.

No obstante, el Confis no tuvo en cuenta la situación deficitaria del Gobierno, por lo que dicha política carecería de impacto en tanto que los recursos con los que cuenta el Gobierno son absolutamente escasos.

\section{Correspondencia de mejores respuestas ${ }^{22}$ - Escenario 1}

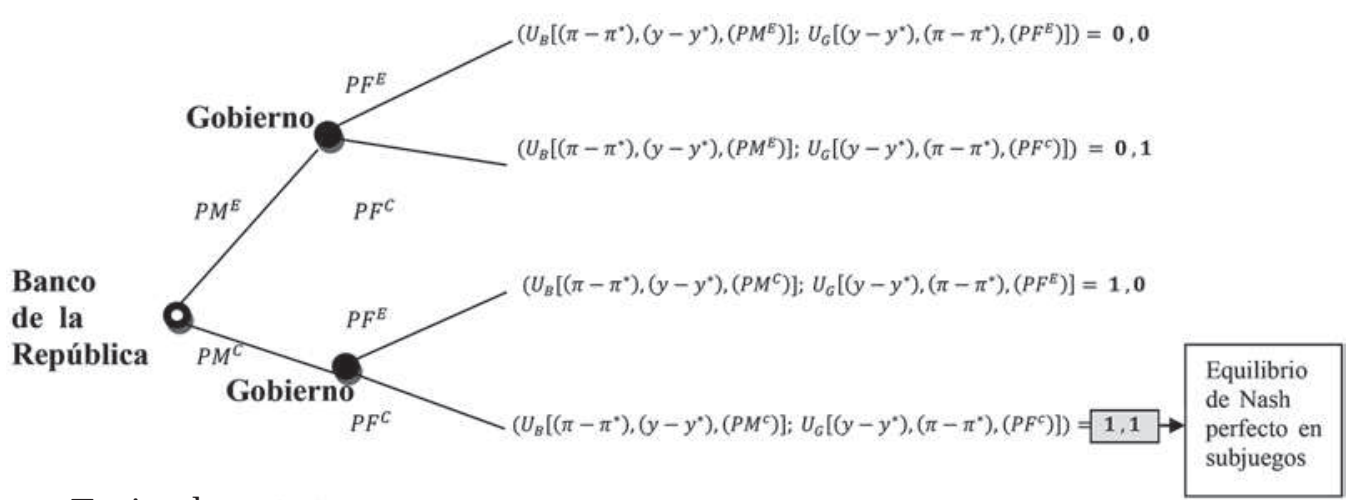

Teniendo en cuenta que,

$P M^{E}$ : Realizar Política Monetaria Expansiva

$P M^{C}$ : Realizar Política Monetaria Contractiva

Por parte del Banco de la República, y por parte de Gobierno Nacional:

$P F^{E}$ : Política Fiscal Expansiva

$P F^{C}$ : Política Fiscal Contractiva

Para la mejor estrategia $\left(P M^{C}\right)$ del Banco de la República correspondiente al escenario 1: $U_{G}\left(P M^{C}, P F^{C}\right), U_{G}\left(P M^{C}, P F^{E}\right)=1,0$

La mejor respuesta del Gobierno ante la estrategia $\left(P M^{C}\right)$ del Banco se da en:

$\operatorname{RG}\left(P M^{C}\right)=P F^{C}$

22. Los valores asignados corresponden a: 1 cuando la estrategia es la acertada teóricamente dado el escenario, y 0 cuando la estrategia no lo es. 
Como es obvio, la mejor respuesta del Banco ante la estrategia $\left(P F^{C}\right)$ del Gobierno está dada en:

$\operatorname{RB}\left(P F^{E}\right)=P M^{E}$

\section{Correspondencia de mejores respuestas - Escenario 2}

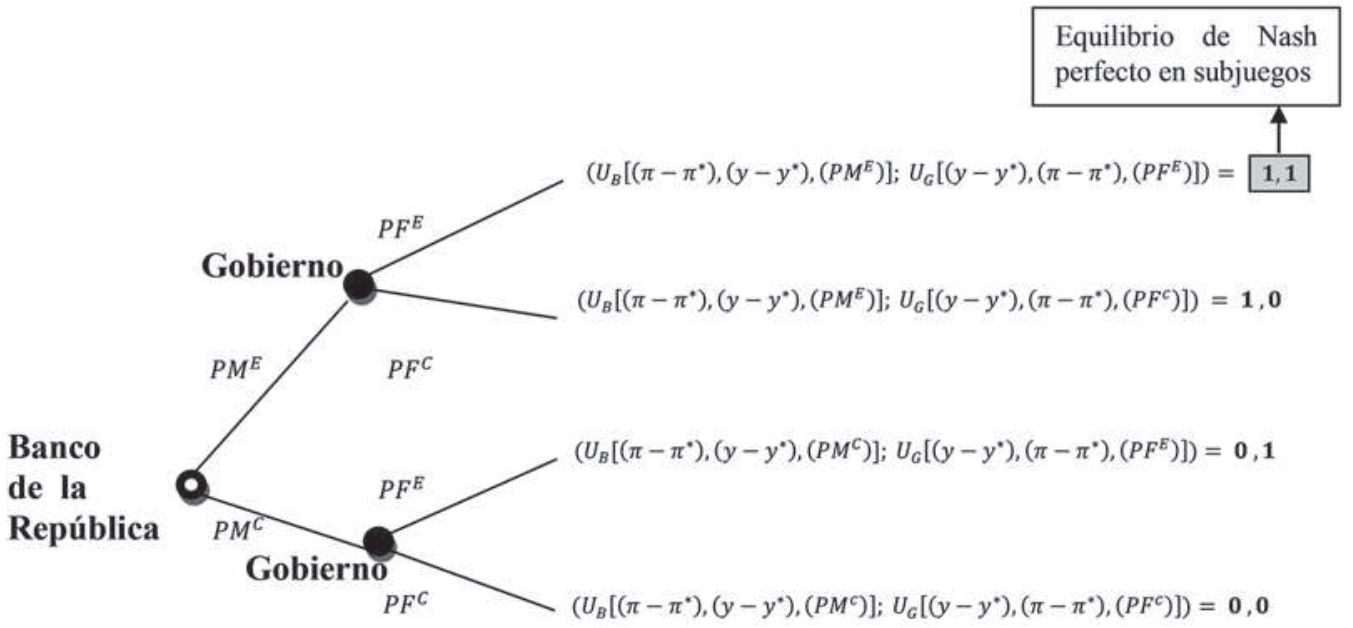

Para la mejor estrategia $\left(P M^{E}\right)$ del Banco de la República correspondiente al escenario 2:

$$
U_{G}\left(P M^{E}, P F^{E}\right)>U_{G}\left(P M^{E}, P F^{C}\right)=1,0
$$

La mejor respuesta del Gobierno ante la estrategia $\left(P M^{E}\right)$ del Banco se da en:

$$
\operatorname{RG}\left(P M^{E}\right)=P F^{E}
$$

Mientras que el Banco tiene como mejor respuesta ante la estrategia $\left(P F^{E}\right)$ del Gobierno:

$$
\operatorname{RB}\left(P F^{E}\right)=P M^{E}
$$

Escenario 3. A partir del análisis de este escenario se pudo establecer el equilibrio de Nash perfecto en subjuego en la interacción de las políticas monetaria expansiva y fiscal expansiva, mediante la ejecución de las siguientes acciones: por parte del Banco de la República una disminución del encaje bancario, mientras que el Gobierno adopta un incremento del gasto público a través de concesión de subvenciones, particularmente al sector exportador.

Tanto el Banco de la República como el Gobierno, en las sesiones simuladas, tomaron las mejores decisiones obteniendo así los más altos beneficios posibles dado el escenario planteado. 


\section{Correspondencia de mejores respuestas - Escenario 3}

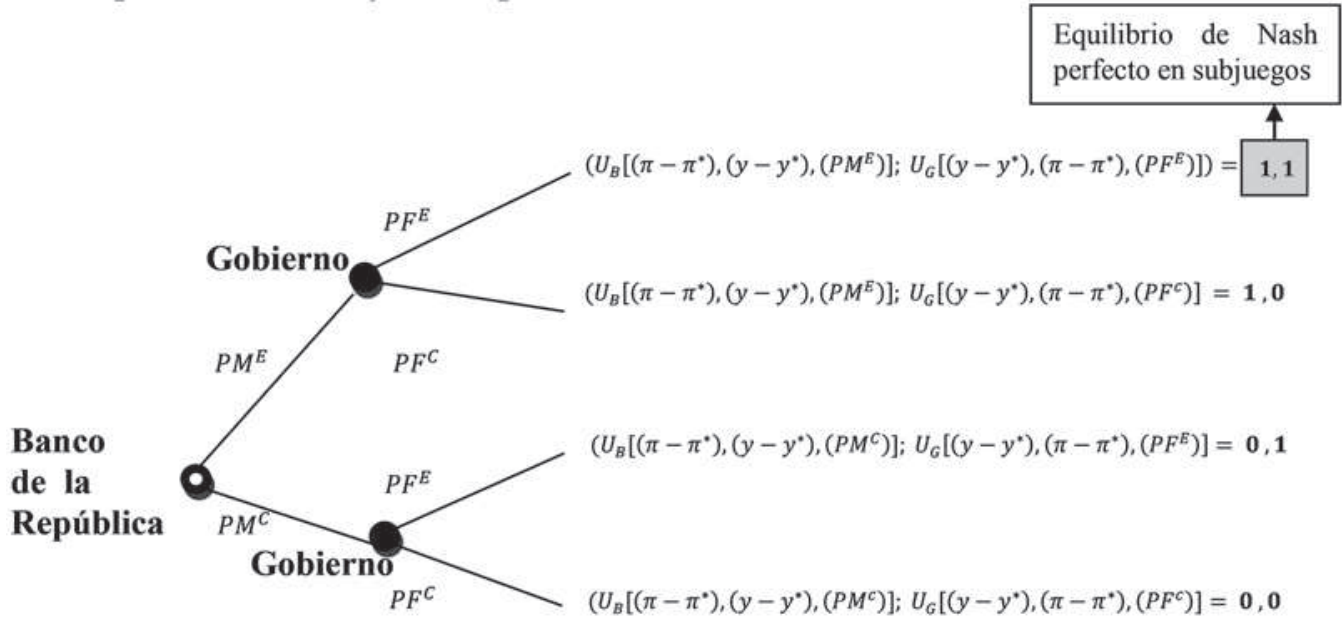

Para la mejor estrategia $\left(P M^{E}\right)$ del Banco de la República correspondiente al escenario 2:

$$
U_{G}\left(P M^{E}, P F^{E}\right)>U_{G}\left(P M^{E}, P F^{C}\right)=1,0
$$

La mejor respuesta del Gobierno ante la estrategia $\left(P M^{E}\right)$ del Banco se da en:

$$
\operatorname{RG}\left(P M^{E}\right)=P F^{E}
$$

En tanto que el Banco tiene como mejor respuesta ante la estrategia $\left(P F^{E}\right)$ del Gobierno:

$$
\operatorname{RB}\left(P F^{E}\right)=P M^{E}
$$

Escenario 4. En este escenario las estrategias que generan mayores beneficios son una política monetaria expansiva a través de las acciones complementarias compra de dólares, compra de TES y disminución del encaje bancario, y una política fiscal contractiva mediante una disminución del gasto público representada en una disminución de las monetizaciones a través de una reducción de la emisión de deuda en moneda extranjera.

En la sesión simulada del ejercicio, la Junta Directiva del Banco de la República optó por, además de comprar divisas, comprar títulos (TES) y disminuir el encaje bancario, en tanto que los agentes de la Junta del Confis asesoraron al Gobierno en el sentido de que aumentara el gasto público.

$\mathrm{Al}$ parecer, en el ejercicio de simulación, la Junta simulada del Confis razonó sobre la conveniencia de aumentar el gasto de tal manera que se pudiera reactivar sectores de la economía doméstica, sin embargo no tuvo en cuenta que el G venía realizando gastos a través de la emisión de deuda, lo que iba en contraposición 
con las decisiones tomadas posteriormente orientadas a disminuir las presiones revaluacionistas.

Es decir, se hacía necesario que dichas decisiones fueran complementadas con la disminución de las monetizaciones, a fin de mejorar las condiciones para el sector exportador - principal perjudicado- y contribuir con el objetivo del BR de mantener inalterada la meta de inflación.

\section{Correspondencia de mejores respuestas - Escenario 4}

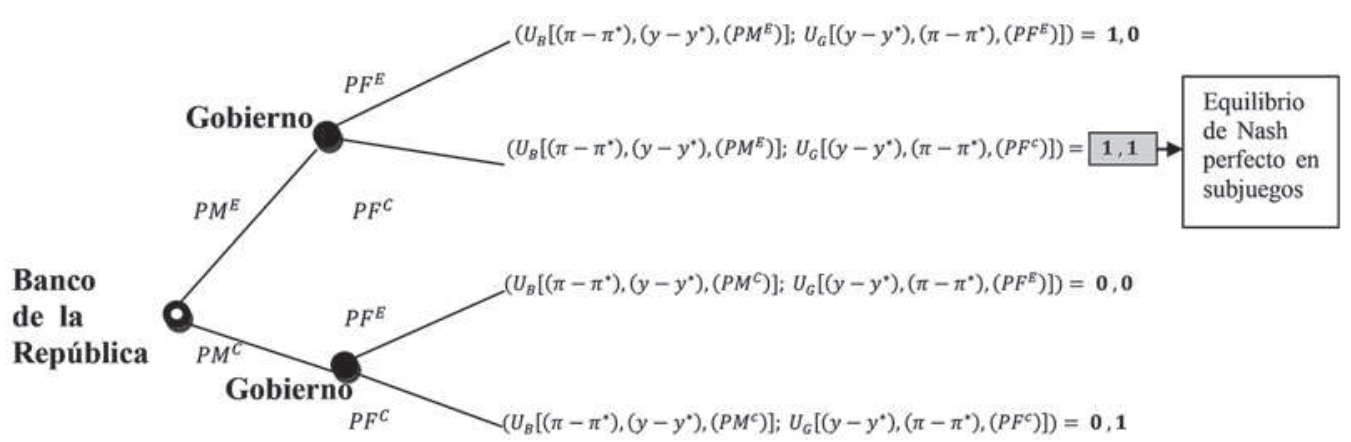

Para la mejor estrategia $\left(P M^{E}\right)$ del Banco de la República correspondiente al escenario 4:

$$
U_{G}\left(P M^{E}, P F^{C}\right)>U_{G}\left(P M^{E}, P F^{E}\right)=1,0
$$

La mejor respuesta del Gobierno ante la estrategia $\left(P M^{E}\right)$ del Banco se da en:

$$
\operatorname{RG}\left(P M^{E}\right)=\left(P F^{C}\right)
$$

La mejor respuesta del Banco ante la estrategia $\left(P F^{C}\right)$ del Gobierno se da en:

$\mathrm{RB}\left(P F^{C}\right)=\left(P M^{E}\right)$

Los resultados del ejercicio de simulación revelan principalmente que las interacciones estratégicas de política económica entre los jugadores Banco de la República y Gobierno Nacional, se encuentran condicionadas por el contexto económico imperante, lo que en última instancia demuestra que no existe una estrategia interactiva evolutiva e intertemporalmente constante, sino que el comportamiento de las variables reales y nominales de la economía tienen un grado de incidencia considerable, sin mencionar la influencia que pueden ejercer las actitudes y personalidades de los individuos decisores de política.

En otras palabras, lo que el ejercicio permitió corroborar es que para alcanzar sus objetivos misionales, el Banco de la República y el Gobierno hacen uso de herramientas diferentes en cada período, dependiendo del contexto económico y político. Los escenarios propuestos en este trabajo dan cuenta de contextos disímiles en donde los jugadores se enfrentaron a situaciones que pusieron a prueba su 
racionalidad económica a fin de maximizar sus beneficios, por lo que en una circunstancia imperó una interacción dinámica entre las estrategias política monetaria contractiva y política fiscal contractiva, en otras una política monetaria expansiva y una política fiscal expansiva, mientras que en otra, se impuso la interacción política monetaria expansiva-política fiscal contractiva. Es decir, que no existe una estrategia que intertemporalmente genere mayores pagos que otras, sino que para cada período, las decisiones de política económica se encuentran condicionadas por los factores mencionados anteriormente.

El ejercicio de simulación muestra que, aunque las decisiones de política del Gobierno fueron tomadas en momentos en los que éste contaba con información completa y perfecta sobre las decisiones que el Banco había adoptado, sólo en el tercer escenario las acciones determinadas por ambas autoridades coincidieron en una instancia de mejores respuestas, constituyendo un equilibrio de Nash perfecto en subjuego.

\section{CONCLUSIONES}

Las relaciones dinámicas entre crecimiento económico e inflación han sido ampliamente estudiadas, lo que se ha visto reflejado en la formulación de teorías y modelos económicos capaces de modelar escenarios particulares que posteriormente son propuestos como generalizaciones, a fin de construir pautas de comportamiento que permitan una acertada intervención de los agentes decisores de la política económica.

En este sentido, el análisis realizado a partir de las simulaciones de sesiones de trabajo de los decisores de la política monetaria y la política fiscal, ha permitido establecer qué acciones ejecutadas por los miembros de la Junta Directiva del Banco de la República y de la Junta del Confis, van dirigidas a mejorar las condiciones de la economía, garantizando así bajas tasas de inflación y un mayor crecimiento del producto, y en consecuencia estabilidad económica.

En particular, las simulaciones dan cuenta de la racionalidad maximizadora de los agentes que intervinieron en el ejercicio, dado que fueron capaces de elegir, dentro de un conjunto de acciones, aquellas a las cuales asignándoles criterios maximizadores les reportaban mayores beneficios, tanto a nivel individual, como a nivel agregado; es decir, que las preferencias de los agentes hacia una u otra acción o estrategia, se dirigieron manifiestamente de acuerdo a los supuestos del homo economicus. Sin embargo, resultó evidente que existe cierta discrepancia entre lo que cada uno de los individuos considera como racional y lo que las decisiones sociales tomadas permiten apreciar como una conducta irracional, dado que los comportamientos de los agentes que intervinieron en las sesiones experimentales en algunos casos resultaron equívocos.

En ese mismo sentido, las simulaciones se realizaron bajo ciertos entornos que delimitaban el comportamiento de los agentes y a los cuales estos últimos debían adaptarse, no obstante, las elecciones de los individuos que conformaban la Junta del Confis estaban condicionadas tanto por los escenarios previamente planteados, como por los escenarios que se configuraban a partir de las decisiones tomadas en el primer momento por la Junta Directiva del Banco de la República, lo que 
sin embargo no supuso irracionalidad individual, pero que sí dejó definido que el Banco como jugador líder condicionaba las decisiones del Confis, aun cuando este último contaba con relativa ${ }^{23}$ ventaja al poseer información completa y perfecta.

En consecuencia, se estableció que la influencia de factores exógenos a los individuos y factores asociados a su personalidad (conocimiento del tema, tolerancia frente a las posturas ajenas, grado de asimilación de consecuencias de actuaciones pasadas, sentimientos morales que priman sobre el interés propio, etc.), determinaron de alguna manera su comportamiento y la forma en que tomaron las decisiones conjuntas, con criterios racionales maximizadores que les permitían asignarle probabilidades subjetivas a las utilidades esperadas de cada acción del conjunto de opciones.

Las interacciones presentadas entre los individuos de cada junta simulada llevaron a la toma de decisiones teóricamente irracionales en conjunto en tres de los escenarios propuestos, lo que condujo a situaciones de no equilibrio de Nash perfectos en subjuegos, debido fundamentalmente a que algunos de los agentes tuvieron percepciones erradas de los beneficios individuales y por supuesto sociales, que les generarían las acciones que estimaron más convenientes, acertadas y pertinentes ante cada escenario propuesto.

De acuerdo con todo lo expuesto arriba, la principal conclusión a la que se llega luego del análisis de los resultados es que, a pesar de la relevancia que tiene el crecimiento económico para el Gobierno, en la mayoría de los casos éste actúa cooperativamente, en concordancia con la ley colombiana y con lo expuesto por Junguito (1994:133), en el sentido de que, en caso de presentarse un conflicto en la coordinación de políticas económicas entre el Banco de la República y el Gobierno, la decisión que se toma siempre es la que más favorece el control de la inflación, sobre todo porque ésta es considerada por los decisores de la política económica, una condición sine qua non para alcanzar un crecimiento sostenido del producto. En ese mismo sentido, se pudo interpretar que para adoptar una u otra acción o estrategia, tanto el Banco como el Gobierno tienen en cuenta el comportamiento futuro esperado de las variables que determinan la estabilidad de sus políticas.

En suma, a partir de la interacción entre la política monetaria y la política fiscal, es posible encontrar situaciones en las que se obtengan equilibrios perfectos en subjuegos en el sentido de Nash, sin embargo, ni los resultados ni la metodología empleada en esta investigación permiten establecer con precisión el nivel óptimo de inflación, ya que éste se encuentra en un rango dentro de cuyos márgenes (en caso de que la meta anual llegare a cumplirse) la tasa es dinámica y depende en gran medida de la evolución de la economía. No obstante, si los comportamientos de los individuos observados durante las sesiones experimentadas corresponden a la conducta de agentes racionales cuyas decisiones se ven afectadas por el entorno y por sus personalidades, entonces resultaría válido afirmar que ante escenarios

23. La teoría económica ha mostrado que el seguidor en este tipo de juegos es el que tiene desventaja. 
tales como la Junta Directiva del Banco de la República y el Gobierno Nacional, en los que los individuos se enfrenten a un conjunto de acciones, de las cuales deben elegir la más estratégica entre todas, sus decisiones estarán determinadas por las decisiones de los otros de acuerdo a sus propios criterios maximizadores de la utilidad.

La independencia del Banco de la República demuestra ser la clave para alcanzar la estabilidad en el nivel de precios y la disciplina fiscal que adicionalmente asume el Gobierno. En este sentido, es evidente que la sociedad puede sufrir mayores pérdidas cuando se amplía la brecha en el nivel de inflación que cuando aumenta la brecha del producto, por lo que generalmente, ante una perturbación negativa en la oferta o la demanda agregadas, la autoridad monetaria tiende a actuar de manera más conservadora, aunque esto no implica necesariamente que lo haga de forma pasiva y puede que actúe de forma tal que contribuya a mejorar los escenarios de la autoridad fiscal. En otras palabras, los acuerdos institucionales entre las autoridades de política permiten que éstas lleguen a instancias donde fijan metas comunes que, a través de la ejecución de acciones individuales, alivian los sesgos de los puntos óptimos de la economía de país.

De modo que, tal como lo sugiere Vargas (1994), cuando el Banco se encuentra en una posición ventajosa (actúa antes que el Gobierno) porque supone que sus decisiones condicionan las decisiones del Gobierno, el resultado más probable que se dé en la economía es la cooperación entre las dos autoridades de política, en el cual ambas maximizan, aunque de manera individual, la función objetivo del Banco. La explicación no se aleja mucho de las conclusiones de Roubini y Sala-iMartin (1992) y Fischer (1993) cuando sugieren que la inflación es una condición para lograr el crecimiento del producto, por lo que los gobiernos la consideran la variable más relevante de la estabilidad macroeconómica, y en consecuencia deciden en función del objetivo de la autoridad monetaria.

Finalmente, a partir de este trabajo se sugieren tres extensiones posibles que podrían complementar la discusión sobre la existencia de equilibrios en el sentido de Nash entre las mejores estrategias de política monetaria y política fiscal, haciendo uso de las técnicas que ofrece la economía experimental. En este sentido se propone i) incluir en el análisis un tercer jugador que represente los intereses de la sociedad, de modo que las decisiones de política económica incorporen las expectativas que el público se genera frente al comportamiento de la economía, ii) establecer las conclusiones a través de la modelación de los resultados con software informático a fin de llegar a reflexiones mucho más precisas y estadísticamente significativas, y iii) diseñar una serie de experimentos (específicamente de la forma de juegos repetidos) en los cuales intervengan individuos con escaso conocimiento sobre el manejo de la política económica, de manera tal que se pueda establecer si dichos individuos actúan de manera racional, lo que conduzca en última instancia a la construcción de modelos empíricos sobre comportamiento estratégico. 


\section{REFERENCIAS}

AHMED, A. \& SALAS, O. (2007). Cooperación y confianza: análisis comparativo en economía experimental. Universidad Santo Tomás. Revista CIFE.

ARÉVALO, J. (2006). Negociación Nash Gradual con Agenda Endógena: un Modelo Trayectoria-Dependiente. Universidad Externado de Colombia.

AUMANN, R. (2006). Guerra y Paz. Revista Asturiana de Economía - RAE, № 36.

BARRO, R \& GORDON, D. (1983). Una teoría positiva de política monetaria en un modelo de tasa natural. Journal of political economic. Vol. $91, \mathrm{~N}^{\circ} 4$.

BENAVIDES, O. \& CÁRDENAS, A. (2005). Relaciones Dinámicas entre crecimiento económico e inflación. Memoria de Grado. Universidad Externado de Colombia.

BERTRAND, J. (1883). Théorie des Richesses: revue de Théories mathématiques de la richesse sociale par Léon Walras et Recherches sur les principes mathématiques de la théorie des richesses par Augustin Cournot. Journal des savants, septiembre, 499-508. Disponible en http://cruel.org/econthought/texts/ marginal/bertrand83.pdf

BILBAO, J. et al. (2000). Avances en teoría de juegos con aplicaciones económicas y sociales. Universidad de Sevilla.

BRANCH, W.; DAVIG, T. \& MCGOUGH, B. (2008). Monetary-Fiscal policy interactions under implementable monetary policy rules (Interacciones entre las políticas monetaria y fiscal implementadas en las reglas de política monetaria). Journal of Money, Credit and Banking, Vol. 40, No. 5. The Ohio State University.

BRAVO, J. (1996). Historia de las matemáticas:Teoría de Juegos. Universidad Autónoma de Madrid, p. 10.

CADAVID, J. (2003). Evolución de la Curva de Phillips en Colombia. Universidad EAFIT. Ecos de Economía. $\mathrm{N}^{\circ} 17, \mathrm{pp} .9-30$.

CANZONERI, M.; CUMBY, R. \& DIBA, B. (2006). Monetary and fiscal policy coordination when bonds provide transactions services (Coordinación de la política fiscal y monetaria cuando se proporcionan servicios de transacción). Georgetown University.

CÁRDENAS, J.; ARANGO, S. \& OLAYA, Y. (2009). Economía experimental en la toma de decisiones en ambientes dinámicos y complejos: una revisión de diseños y resultados. Pontificia Universidad Javeriana. Cuadernos de Administración. Vol. 22. № 23, pp. 31-57.

COURNOT, A. (1838). Researches into the mathematical principles of the Theory of Wealth. Londres: MacMillan Company. Disponible en: http://www3.nd.edu/tgresik/IO/Cournot.pdf

CHAMBERLIN, E. (1933). Theory of monopolistic competition. Cambridge, Massachusetts: Harvard University.

CLAVIJO, S. (2000). Banca central y coordinación macroeconómica: El caso de Colombia. Banco de la República.

CRAWFORD. V. (2000). John Nash and the analysis of strategic behavior (John Nash y el análisis del comportamiento estratégico). University of California, San Diego. Department of Economics. 
DE GREGORIO, J. (1996). Inflación, crecimiento y bancos centrales: teoría y evidencia empírica. Una versión anterior de este trabajo fue presentada en la ciudad de Bogotá, durante el Seminario Latinoamericano de Crecimiento Económico organizado por el Gobierno de Colombia, el Banco Mundial y el BID.

DONAYRE, L. \& GONZÁLEZ, A. (2002). Hacia la coordinación de políticas: una perspectiva dinámica basada en juegos diferenciales. Banco Central de Reserva del Perú.

Epstein, R. y Rubinfeld, D. (2001). Merger Simulation: A simplified approach with new applications. Antitrust Journal, 69, 883-919. Disponible en http://scholarship.law.berkeley.edu/cgi/viewcontent.cgi?arti cle $=2362 \&$ context $=$ facpubs

FISCHER, S. (1993). The role of Macroeconomic factors in growth. (El papel de los factores macroeconómicos en el crecimiento). NBER Working Paper.

FLOOD, M. (1958). Some experimental games (Algunos juegos experimentales). Management Science 5, pp. 11-17.

FRÉCHET, M. (1958). Las matemáticas y lo concreto. Press Universitaires de France. Traducción Universidad Nacional Autónoma de México. Dirección General de Publicaciones.

GIBBONS, R. (1992). Un primer curso de teoría de juegos. Ed. Antoni Bosch. Barcelona.

GONZÁLEZ, J. (1999). Ajuste macro, política monetaria y empleo. Nómadas, pp. 70-85.

GUTIÉRREZ, J.; GUZMÁN, C. \& JIMÉNEZ, U. (2000). Economía política y finanzas públicas: teoría, evidencia y resultados de laboratorio. Revista de Economía Institucional. Universidad Externado de Colombia.

HAERINGER, G. (2004). Teoría de los juegos y de las decisiones. Curso académico: 2003-2004.

HEIFFINGER, S. \& HAAN, J. (1996). The Political Economy of Central-Bank Independence (La política económica de un Banco Central Independiente). Center for Economic Research.

JUNGUITO, R. (1994). La independencia de la banca central en América Latina. Borradores de Economía, Banco de la República, p. 133.

KALMANOVITZ, S. (1999). La política fiscal colombiana en un contexto histórico. Borradores de Economía, Banco de la República.

KUNDER, P. (2000). Introducción a la teoría del grado topológico de la aplicación. Ed. Uninorte.

LAMBERTINI, L. \& DIXIT, A. (2001). Monetary-fiscal policy interactions and commitment versus discretion in a monetary unión (Interacción de políticas monetarias y fiscales y el compromiso frente a discreción en una unión monetaria). University Princeton.

LAMBERTINI, L. \& ROVELLI, R. (2004). Monetary and fiscal policy coordination and macroeconomic stabilization. A theoretical analysis (La coordinación de la política fiscal y monetaria y la estabilización macroeconómica. Un análisis teórico). Dipartimento di Scienze Economiche Università di Bologna.

LÓPEZ, C. (2006). Equilibrio de reputación y credibilidad: una modelación simple. UNAM.

LÓPEZ, M. (2007). Algunos descubrimientos matemáticos del siglo XX. Real Academia de Ciencias Exactas, Físicas y Naturales de España. VIII Programa de Promoción de la Cultura Científica y Tecnológica. Vol. 101, No. 2, pp. 285-305.

LOZANO, L. \& HERRERA, M. (2008). Dominancia fiscal versus dominancia monetaria: evidencia para Colombia, 1990-2007. Unidad de Investigaciones Económicas del Banco de la República.

MARTINELLI, C.; GASCO, L. (2006). Economía dinámica, economía aplicada y teoría de juegos. Ensayos en Homenaje a Ramón García-Cobián. Pontificia Universidad Católica del Perú.

MONSALVE, S. (2002). Teoría de juego: ¿Hacia dónde vamos? (60 años después de von Neumann y Morgenstern). Revista de Economía Institucional, Vol. 4, № 7. Segundo semestre. 
MONSALVE, S. (2003). John Nash y la teoría de juegos. Universidad Nacional de Colombia. Lecturas Matemáticas. Vol. 24, pp. 137-149.

MONSALVE, S. \& ARÉVALO J. (2006). Un curso de la teoría de juegos clásica. Universidad Externado de Colombia, p. 16.

NASH, J. (1950). Non-cooperative games (Juegos no cooperativos). Princeton University. Department of mathematics.

NICHOLSON, W. (2007). Teoría Microeconómica. Principios básicos y aplicaciones. Novena edición, p. 536.

PARRA, F. (2012). Experimentos económicos como herramientas de aprendizaje: caso del juego de bienes. Universidad Industrial de Santander. Escuela de Economía y Administración.

ROUBINI, N. \& SALA-I-MARTIN, X. (1992). A growth model of inflation, tax evasion, and financial repression (Un modelo de crecimiento de la inflación, la evasión fiscal, y la represión financiera). NBER Working Paper No. 4062.

SARGENT,T. \&WALLACE, N. (1980). Some unpleasant monetarist arithmetic (Algo de incómoda aritmética monetaria). University of Minnesota.

SHAPIRO, C. (1996). Mergers with Differentiated Products. Department of Justice. Working Paper.

SMITH, V. (1994). Economics in the laboratory (Economía en el laboratorio). Journal of Economic Perspectives. Vol. 8. N 1, pp. 113-131.

TOBIN, J. (1966). "The cruel dilemma". Price issues in theory, practice, and policy ("El cruel dilema". Los problemas de los precios en teoría y práctica, y la política económica). University of Pennsylvania.

VARGAS, H. (1994). ¿A qué juegan el gobierno y un banco central independiente? Borradores Semanales de Economía. Subgerencia de Estudios Económicos del Banco de la República.

JEHLE, G. \& PHILIP, R. (2001). Advanced microeconomic theory. Ed. Addison Wesley.

VON NEUMANN, J. \& MORGENSTERN, O. (1944). Theory of games and economic behavior (Teoría de juegos y comportamiento económico). Princeton University Press.

VON STACKELBERG, H. (1934). Market structure and equilibrium. Vienna: Springer Verlag.

WOODFORD, M. (1995). Price level determinacy without control of a monetary aggregate (Determinismo sin control del nivel precios de un agregado monetario). NBER. Working Paper Series.

WERDEN, G. (1997). Simulating the effects of differentiated products mergers: a practical alternative to structural merger policy. George Mason Law Review, 5, 363-386. 